\title{
Physicians' Attitudes Toward Guidelines for Stroke: A Survey of Korean Neurologists
}

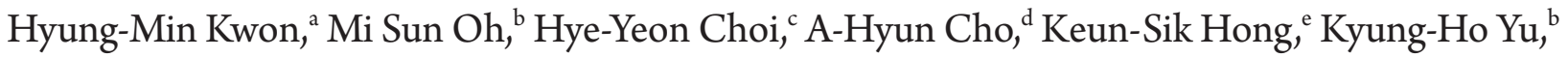 \\ Hee-Joon Bae, ${ }^{\mathrm{f}}$ Juneyoung Lee, ${ }^{\mathrm{g}}$ Byung-Chul Lee ${ }^{\mathrm{b}}$ \\ ${ }^{a}$ Department of Neurology, Boramae Medical Center, Seoul National University College of Medicine, Seoul, Korea \\ ${ }^{b}$ Department of Neurology, Hallym University Sacred Heart Hospital, Hallym University College of Medicine, Anyang, Korea \\ 'Department of Neurology, Gangdong Kyung Hee University Hospital, Kyung Hee University College of Medicine, Seoul, Korea \\ 'Department of Neurology, Yeouido St. Mary's Hospital, The Catholic University of Korea, College of Medicine, Seoul, Korea \\ eDepartment of Neurology, Ilsan Paik Hospital, Inje University, Goyang, Korea \\ ${ }^{\mathrm{f}}$ Department of Neurology, Seoul National University Bundang Hospital, Seoul National University College of Medicine, Seongnam, Korea \\ 'Department of Biostatistics, Korea University College of Medicine, Seoul, Korea
}

Background and Purpose Clinical practice guidelines (CPGs) are regarded as an essential guidance tool for practicing physicians. We surveyed physicians in Korea in order to evaluate their attitudes toward the Korean CPGs for stroke.

Methods We obtained participation agreement for our survey from 27 centers of the 33 most actively contributing to the Korean Stroke Registry. A total of 174 neurologists participated in a questionnaire interview regarding their attitudes toward CPGs for stroke.

Results Of 174 participating neurologists, 65 (37.4\%) were stroke neurologists. The average age was $33.6 \pm 7.1$ and $49(28.2 \%)$ were female. Most of the respondents held positive attitudes and opinions regarding the use of the guidelines, whereas only a small percentage $(14.9 \%)$ responded negatively. More than $60 \%$ of the physicians in the survey reported adherence to the Korean CPGs in dyslipidemia management for the secondary prevention of stroke.

Conclusions The positive attitudes and opinions toward the guidelines imply that physicians' attitudes should not be regarded as a potential barrier to the implementation of Korean CPGs for stroke practiced by general physicians.

\author{
Correspondence: Byung-Chul Lee \\ Department of Neurology, Hallym \\ University Sacred Heart Hospital, \\ Hallym University College of Medicine, \\ 22 Gwanpyeong-ro 170beon-gil, \\ Dongan-gu, Anyang 431-070, Korea \\ Tel: +82-31-380-3741 \\ Fax: +82-31-381-4659 \\ E-mail: ssbrain@hallym.ac.kr
}

Received: January 13, 2014 Revised: March 26, 2014 Accepted: April 8, 2014

This study was sponsored by Pfizer Pharmaceuticals Korea Ltd.

The authors have no financial conflicts of interest.

Keywords Guideline; Stroke; Attitude

\section{Introduction}

Clinical practice guidelines (CPGs) are systematically developed statements aimed at helping practitioners and patients make informed health care decisions in specific clinical circumstances. ${ }^{1}$ Investigators of the Clinical Research Center for Stroke (CRCS), funded by the Ministry of Health and Welfare of Korea, developed and published the first edition of the Korean CPGs for stroke in 2009, and have since updated the guidelines to reflect and incorporate new evidence pertinent to clinical practice. The Korean CPGs for stroke have been endorsed by relevant and respected academic societies and have been distributed in the forms of monographs, summary manuals, freely accessible PDF files on the websites of the CRCS and the Korean Stroke Society, and through smart phone applications in order to ensure widespread dissemination and implementation.

CPGs, which help incorporate scientific advancements into daily practice, are expected to improve the quality of care, lead 
to better patient outcomes, avoid unnecessary cost, and serve as good educational tools. ${ }^{2}$ However, physicians' attitudes toward CPGs, as well as their confidence in the instruments, are essential for successful implementation and physician adherence. ${ }^{3}$ Since the publication of the Korean CPGs for stroke, the attitudes and confidence levels of Korean neurologists toward the guidelines have not been explored.

\section{Methods}

This study was designed a priori as a sub-study within the Real world of Lipid-Lowering therapy in Korean Stroke patients (ROLLERKOST), which aimed to assess Korean neurologists' knowledge of current dyslipidemia management guidelines and guideline-based discharge prescriptions for statin amongst patients hospitalized with acute ischemic stroke or transient ischemic attack. Details of the ROLLERKOST study have already been published, ${ }^{4}$ but to summarize, 33 centers that actively enroll their patients in the Korean Stroke Registry were selected from a total of 86 neurology training hospitals in Korea. ${ }^{5}$ These 33 centers were sent an e-mail describing the purpose of the study and requesting their participation. Consent was received from 27 centers. Between November 2010 and December 2011, we conducted a survey that directly interviewed neurologists (boardcertified neurologists and residents) from the 27 centers, using a structured questionnaire composed of four main principles: physician characteristics (5 items), practice patterns of dyslipidemia management (15 items), knowledge of the current dyslipidemia management guidelines (10 items) and attitudes to and confidence in the current guidelines ( 21 items).

The current study analyzed responses to the 21 questions on physicians' attitudes towards and confidence in the current Korean CPGs for stroke (Table 1). We used a 5-point Likert scale to rate physicians' responses to each of the 21 questions: strongly agree, agree, neither agree nor disagree, disagree, and strongly disagree. The respondents' attitudes were then divided into positive views (strongly agree and agree) and negative views (strongly disagree and disagree). Neutral responses (neither agree nor disagree) were disregarded. The internal consistency of the responses of physicians to the 21 questions was examined using Cronbach's alpha. In measuring the consistency, the responses of items $14,15,16,17,18,20$, and 21 as listed in Table 1 were reversed to ensure that all items had the same direction. Descriptive statistics were used to present the results of physicians' responses to each item. In addition, we investigated which guidelines Korean neurologists usually referred to during their clinical practice of dyslipidemia management (the Korean CPGs, National Cholesterol Education Program-Adult Treatment Panel
Table 1. Items included in the questionnaire

Item
1. Too simplified for use in clinical practice
2. Too strict for use in clinical practice
3. Infringement of physician's autonomy
4. Increased probability of medico-legal suit
5. Developed by less experienced physicians
6. Pressure on physician's decision-making in clinical practice
7. Too inaccessible for use in clinical practice
8. Disagreement with guidelines
9. Treatments following guidelines are likely to be ineffective
10. Difficulty in changing his/her current practice pattern
11. Lack of time to strictly follow guidelines
12. Lack of educational materials
13. Difficulty in using guidelines
14. Developed to improve quality of care
15. Developed to cut down health care costs
16. Developed by experts without any bias
17. Providing expert advice in a convenient way
18. Good educational tool
19. Too complicated for use in clinical practice
20. Have you ever read the dyslipidemia management of the current Korean
Stroke Guidelines?
21. Are you well aware of the dyslipidemia management guidelines for secondary
stroke prevention?

III guidelines, ${ }^{6}$ or the reimbursement guidelines provided by the Korean Health Insurance Review and Assessment Service).

\section{Results}

Of the 33 centers contacted, 27 centers participated in the study (81.8\%). These consisted of 18 university hospitals, 7 affiliated hospitals, and 2 secondary referral hospitals. A total of 174 neurologists who were both actively involved in managing patients and consented to face-to-face interviews responded to the questionnaire. The average age of respondents was 33.6 years $\pm 7.1,49(28.2 \%)$ were female, 73 (42.0\%) were boardcertified neurologists and 66 (37.9\%) indicated that their subspecialty was stroke medicine. Detailed demographic characteristics are presented in Table 2. The internal consistency of responses was relatively high (Cronbach's $\alpha=0.8677$ ).

Most of the respondents reported a positive attitude to the use of CPGs for stroke in their clinical decision-making (Figure 1 ). The mean value of negative views regarding all questionnaires (excluding the statements that were neither positive nor negative) was $14.9 \%$. More than $70 \%$ of the respondents responded that they used the Korean CPGs for stroke and believed that treatments following these guidelines are likely to be effective without infringing on physician autonomy. Only $34 \%$ of the respondents, however, were confident that CPGs for stroke are unbiased statements, and a sizable proportion of re- 
spondents (48.9\%) complained about a perceived lack of education materials, despite a healthy majority of neurologists holding the belief that CPGs for stroke are developed to improve the quality of care afforded to patients and that they are a good educational tool (Figure 1).

In terms of the level of knowledge of current dyslipidemia management guidelines, there was no difference in the attitudes

Table 2. Demographic characteristics of interviewed neurologists $(n=174)$

\begin{tabular}{lc}
\hline Characteristic & Number (\%) \\
\hline Age $(y r)$ & \\
$20-29$ & $70(40.2)$ \\
$30-39$ & $65(37.4)$ \\
$40-49$ & $35(20.1)$ \\
$50-59$ & $4(2.3)$ \\
Gender & \\
Male & $125(71.8)$ \\
Female & $29(28.2)$ \\
Physicians & \\
Residents & $101(58.1)$ \\
Board-certified neurologists & $73(41.9)$ \\
Stroke subspecialty & $66(37.9)$ \\
Years of being a neurologist & \\
$0-1$ & $42(24.1)$ \\
$2-6$ & $64(36.8)$ \\
$7-10$ & $14(8.1)$ \\
$11-15$ & $20(11.5)$ \\
$>15$ & $34(19.5)$ \\
\hline
\end{tabular}

or confidence levels on the distribution of responses between the higher- and lower-knowledge level groups for all questions. The median score for the neurologists' knowledge of the current guidelines was 70 (range, 30-100). A total of 79 (45.4\%) neurologists were thus categorized into the higher-level knowledge group, having achieved a score of $>70$, and 95 (54.6\%) were categorized into the lower-level knowledge group. When between-group responses were compared for specialization (stroke neurologists vs. non-stroke neurologists) and certification (board-certified neurologists vs. residents), stroke neurologists and board-certified neurologists showed significantly higher positive responses ( $3.58 \pm 0.41$ vs. $3.39 \pm 0.40, P=0.0027 ; 3.57 \pm$ 0.42 vs. $3.38 \pm 0.39, P=0.0034$, respectively).

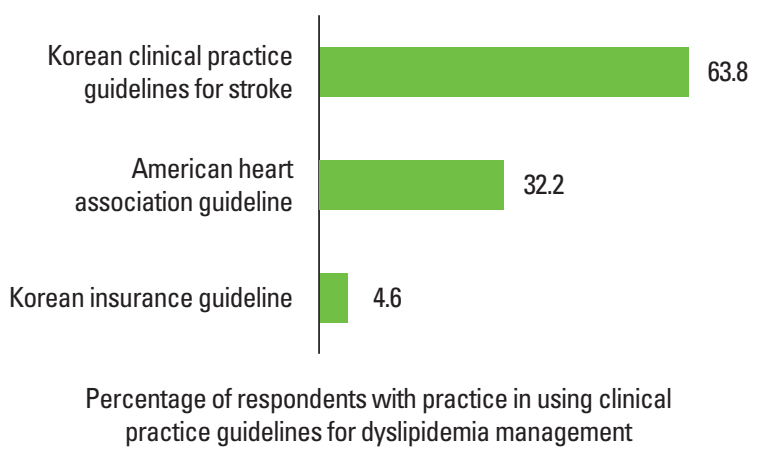

Figure 2. Respondents' work habits in using clinical practice guidelines for dyslipidemia management.

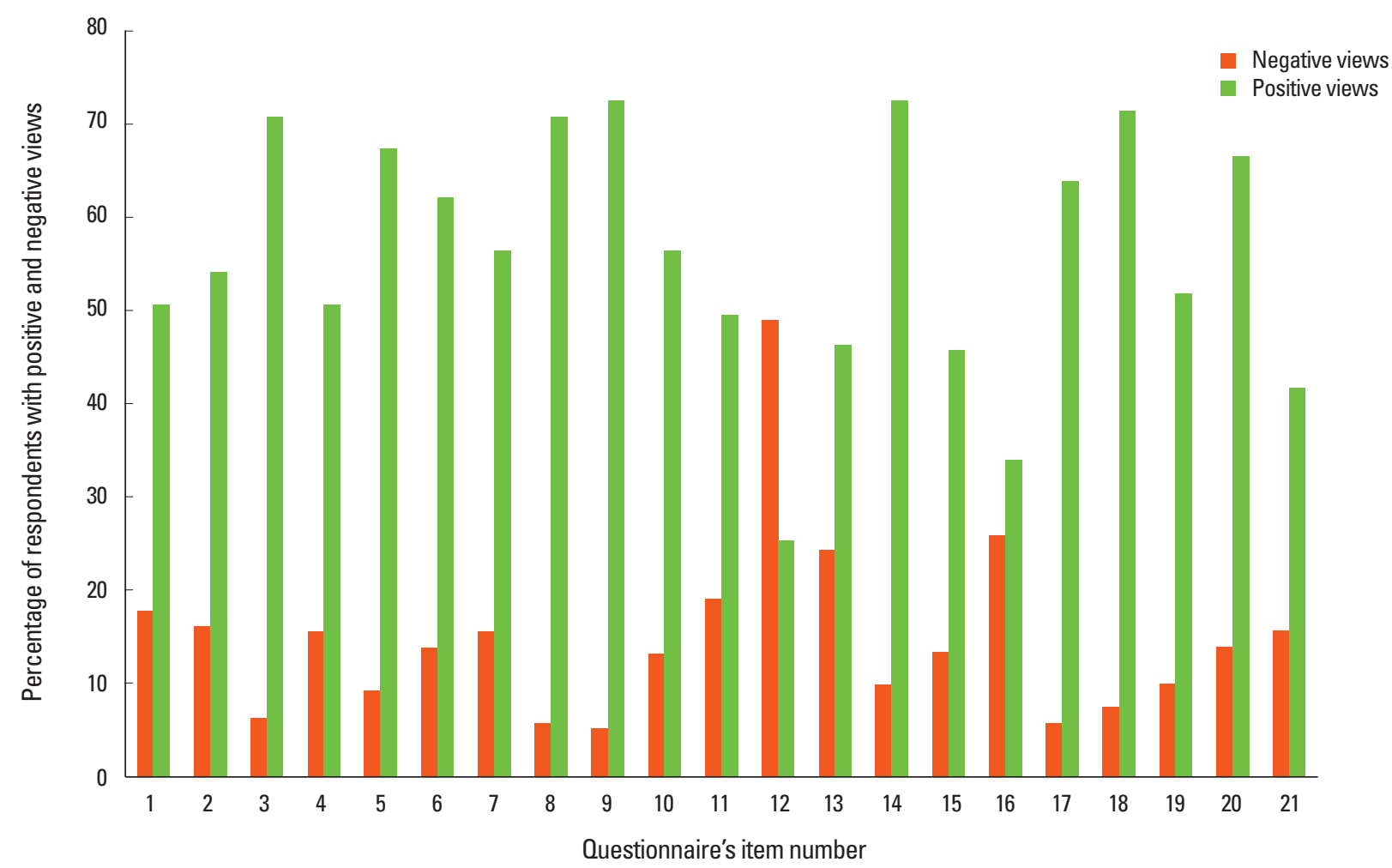

Figure 1. Attitudes and opinions of Korean neurologists toward clinical practice guidelines for stroke. 
Respondents' practical use of CPGs for stroke is presented in Figure 2. It was found that over $60 \%$ of the health care providers in this survey adhered to domestic CPGs during dyslipidemia management (American Heart Association guidelines, 32\%, Korean Health Insurance guidelines, 5\%).

\section{Discussion}

Before designing a strategy for the implementation of CPGs, it is important to explore the attitudes and opinions held by health care professionals. Skeptical opinions negatively influence the implementation of guidance, either directly or indirectly, through the creation of an unfavorable environment characterized by lack of support from peers and superiors. ${ }^{7}$ The positive attitudes and opinions toward CPGs for stroke held by Korean neurologists as reported in this survey, combined with the findings of previous studies among diverse groups of health care professionals, ${ }^{8,9}$ indicate that the current attitudes of physicians do not form a barrier to the future implementation of CPGs for stroke.

Responses to the questionnaire were reasonably consistent for all respondents (Cronbach's $\alpha=0.8677$ ). It was, however, somewhat discouraging that a negative average response to the statement "The specialists' opinions are coordinated without any bias" (item number 16) was obtained, as similar questions such as "They are made by specialists who lack actual experience in medical fields" (item number 5) and "It is hard to agree on the guidelines" (item number 8 ), yielded positive responses (Figure 1). This discrepancy might be explained by the negative phraseology of the statement, but the possibility that this might be a true response cannot be excluded.

Respondents' work habits also concurred with their attitudes and opinions toward CPGs for stroke. A total of $64 \%$ of respondents stated that they follow the treatment advised by the dyslipidemia guidelines, and $66.5 \%$ had already read the dyslipidemia guidelines in the management of stroke patients (Figure 2). This finding might be related to professionals' experience in using CPGs for stroke and a culture of evidence-based practice. Furthermore, specific criteria, such as whether the source of the guidelines is a credible and respected body or organization, encourage health care providers to use and adhere to certain CPGs. ${ }^{10}$ As a result, the fact that our CPGs for stroke have been accredited by the Korean Stroke Society, the Korean Neurological Association, and the Korean Society of Cerebrovascular Surgeons might be one of the more significant reasons for their implementation.

In our previous ROLLERKOST study, Korean neurologists with a higher knowledge level were more likely to adhere to guideline-based discharge prescription of statin. ${ }^{4}$ In this analysis, however, there was no significant difference in the attitudes or confidence on the distribution of responses between the higherand lower-knowledge level groups for all questions. It has not been well demonstrated whether knowledge level is associated with adherence to guidelines in clinical practice. Physicians' increased familiarity with the guidelines and their improved knowledge of them may correlate with a higher rate of adherence to prescription, but there was no association between knowledge level and attitudes toward guidelines for stroke.

To the authors' knowledge, this is the first study in which attitudes towards CPGs for stroke among Korean neurologists have been examined. However, we also admit several methodological limitations. Respondent physicians were affiliated with neurology training hospitals, so this may limit the generalizability of our findings. Moreover, the mean age of physicians was quite low because most respondents were either trainee residents or had only become certified neurologists within the last six years. Further studies should therefore investigate the reasons behind non-adherence following the implementation of specific CPGs among general physicians of a wider age range.

\section{Conclusions}

This study shows that most of the respondents in our survey held positive attitudes and opinions regarding the use of the Korean CPGs for stroke, whereas only a small percentage responded negatively. The positive attitudes and opinions toward the guidelines suggest that physicians' attitudes should not be regarded as a potential barrier to the implementation of Korean CPGs for stroke.

\section{References}

1. Field MJ, Lohr KN, eds. Clinical practice guidelines: directions for a new program. Washington, DC: National Academy Press; 1990.

2. Woolf SH, Grol R, Hutchinson A, Eccles M, Grimshaw J. Clinical guidelines: potential benefits, limitations, and harms of clinical guidelines. BMJ 1999;318:527-530.

3. Cabana MD, Rand CS, Powe NR, Wu AW, Wilson MH, Abboud PA, et al. Why don't physicians follow clinical practice guidelines? A framework for improvement. JAMA 1999;282: 1458-1465.

4. Hong KS, Oh MS, Cho HY, Cho AH, Kwon HM, Yu KH, et al. Statin prescription adhered to guidelines among patients hospitalized due to acute ischemic stroke or transient ischemic attack. J Clin Neurol 2013;9:214-222.

5. Jung KH, Lee SH, Kim BJ, Yu KH, Hong KS, Lee BC, et al. 
Secular trends in ischemic stroke characteristics in a rapidly developed country: results from the korean stroke registry study (secular trends in Korean stroke). Circ Cardiovasc Qual Outcomes 2012;5:327-334.

6. Third report of the National Cholesterol Education Program (NCEP) expert panel on detection, evaluation, and treatment of high blood cholesterol in adults (adult treatment panel III) final report. Circulation 2002;106:3143.

7. Francke AL, Smit MC, de Veer AJ, Mistiaen P. Factors influencing the implementation of clinical guidelines for health care professionals: a systematic meta-review. BMC Med Inform Decis Mak 2008;8:388.
8. Hayward RS, Guyatt GH, Moore KA, McKibbon KA, Carter AO. Canadian physicians' attitudes about and preferences regarding clinical practice guidelines. CMAJ 1997;156:17151723.

9. Quiros D, Lin S, Larson EL. Attitudes toward practice guidelines among intensive care unit personnel: a cross-sectional anonymous survey. Heart Lung 2007;36:287-297.

10. Powell-Cope GM, Luther S, Neugaard B, Vara J, Nelson A. Provider-perceived barriers and facilitators for ischaemic heart disease (IHD) guideline adherence. J Eval Clin Pract 2004;10: 227-239. 